\title{
Acerca de Henry Lewis Morgan (1818-1881) y su obra “Ancient Society"
}

About Henry Lewis Morgan (1818-1881) and his work called Ancient Society

Sobre o Henry Lewis Morgan (1818-1881) e o seu traballho “Ancient Society”

\section{Cristo Manuel Marrero González ${ }^{1}$}

${ }^{1}$ Enfermero en el Servicio Canario de la salud. Área de salud de Tenerife (España). Doctor por la

Universidad de La Laguna (España). Estudiante de Antropología Social y Cultural de la

Universidad Nacional de Educación a Distancia (España).

Cómo citar esta reseña en edición digital: Marrero González, C. (2020). Reseña de Henry Lewis Morgan (1818-1881) y su obra “Ancient Society”. Cultura de los Cuidados (Edición digital), 24(56). Recuperado de http://dx.doi.org/10.14198/cuid.2020.56.24

Marrero González, C.

Correspondencia: Calle Infante Juan Manuel 16, 38010. Santa Cruz de Tenerife. Tenerife. Correo electrónico: cmmarrerogonzalez@gmail.com

Recibido/ Aceptado: Reseña Invitada.

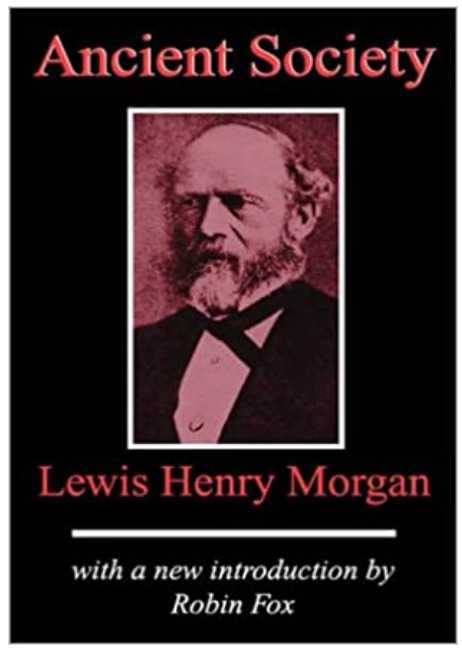

Lewis Henry Morgan (1977). Ancient Society. Barcelona: Icaria. ISBN 10: 84336-0103 


\section{RESUMEN}

En este presente breve ensayo, se esbozan las principales ideas de la obra “Ancient Society”, publicada en 1871, del antropólogo Henry Lewis Morgan (1818-1881). Morgan es considerado uno de los primeros antropólogos en Estados Unidos y enmarcado en la escuela del evolucionismo clásico siendo contemporáneo de otros evolucionistas como Tylor. El autor representa una figura clave en el "renacimiento" de ideas evolucionistas a partir de los años treinta del siglo XX y para influir en la inspiración de la ecología cultural, el materialismo cultural y la antropología del parentesco a partir de la mitad del siglo XX.

Palabras clave: Henry Lewis Morgan, Ancient Society, antropología, evolucionismo clásico.

\section{ABSTRACT}

In this present brief essay, I describe the main ideas of the work "Ancient Society", published in 1871, by the anthropologist Henry Lewis Morgan (1818-1881). Morgan is one of the first anthropologists in the United States. Henry Lewis Morgan is framed in classical evolutionism school and he is contemporary of other evolutionists like Tylor. The author represents a key figure in the "renaissance" of evolutionary ideas from the thirties of the twentieth century to be inspiration of cultural ecology, cultural materialism and the anthropology of kinship from the midtwentieth century.

Key words: Henry Lewis Morgan, Ancient Society, anthropology, classic evolucionism.

\section{RESUMO}

Neste breve ensaio eu escrevo as principais idéias da obra "Ancient Society", publicada em 1871, pelo antropólogo Henry Lewis Morgan
(1818-1881). Morgan é considerado um dos primeiros antropólogos nos Estados Unidos e enquadrado na escola do evolucionismo clássico sendo contemporâneo com outros evolucionistas como Tylor. $\mathrm{O}$ autor representa uma figura chave no "renascimento" das idéias evolucionárias dos anos 30 do século XX. É influencia e inspiração da ecologia cultural, do materialismo cultural e da antropologia do parentesco a partir de meados do século $\mathrm{XX}$.

Palavras-chave: Henry Lewis Morgan, Ancient Society, antropologia, evolucionismo clássico

\section{RESEÑA INTRODUCTORIA Y CONTEXTO HISTÓRICO}

Henry Lewis Morgan (1818-1881), nacido en Nueva York en Estados Unidos de formación jurista es considerado uno de los pioneros de la antropología social. Dentro de la antropología social y cultural se encuentra enmarcado dentro de la escuela del evolucionismo clásico, contemporáneo de otros autores de esta misma escuela como Herbert Spencer o Edward Burnet Tylor. Es a partir de estos autores cuando comienza la andadura de la antropología como disciplina a mitad del siglo XIX al acuñarse por primera vez el término de cultura por Tylor, del uso del método comparativo como herramienta de investigación y por las influencias de la teoría evolucionista. Hay que decir que como antecedente de la Ilustración para las ideas evolucionistas de Morgan y de otros contemporáneos, se 
Revista científica de la Asociación de Historia y Antropología de los Cuidados (Universidad de Alicante)

encuentra Montesquieu al describir las tres etapas por las que evoluciona una sociedad: estado salvaje, estado de barbarie y de civilización. Coincide en que en la época de Morgan se estaba dando la expansión del colonialismo por parte de occidente.

Morgan, Tras haberse graduado en derecho, trabajó como abogado asesorando a los indios de la tribu Séneca acerca del acceso sobre sus tierras lo que le llevó a interesarse por los Iroqueses. Este interés le llevó a publicar el libro "La Liga de los Iroqueses" (1851), donde se analiza la cultura de los indios. Cabe destacar entre otras contribuciones su descubrimiento del sistema clasificatorio de parentesco.

No obstante su mayor contribución a la antropología se encuentra en su obra Ancient Society (1871), donde se presenta un enfoque evolucionista materialista que influenciaría más adelante a autores como Engels, Leslie White o Marvin Harris. Cada etapa evolutiva con ciertos tipos de tecnologías y subsistencias. A continuación, se explican las ideas principales de un extracto de "La Sociedad Antigua" que se encuentran en la obra de Bohannan y Glazer (2007).

\section{ANCIENT SOCIETY (LA SOCIEDAD} ANTIGUA, 1871)

En el presente documento, se abordan los temas relacionados con la evolución de la humanidad de forma unilineal desde el estado más simple de salvajismo, hasta la llamada civilización en cuanto a forma de gobierno, tipos de estructuras de familia, formas de subsistencia y progreso que se da en las etapas evolutivas.

\section{Los períodos étnicos}

Morgan explica cómo la humanidad ha ido evolucionando unilinealmente en varias etapas en cuanto a subsistencia, gobierno, lengua, familia, religión, vida doméstica y arquitectura y en cuanto a propiedad. Para alcanzar la civilización, o culmen evolutivo humano en estas áreas, se ha pasado desde una primera etapa de salvajismo, pasando por un estado de barbarie hasta llegar a la etapa final de civilización. El desarrollo evolutivo en estas etapas se forma gradualmente. De hecho, las civilizaciones contemporáneas tienen origen evolutivo desde su estado de barbarie y que a su vez tomaron origen del estado de barbarie desde el salvajismo. A través de inventos $\mathrm{y}$ descubrimientos y a través de gérmenes de pensamiento la humanidad pasa de una etapa a otra gradualmente.

El período de salvajismo es dividido en tres etapas: estado inferior de salvajismo, estado medio de salvajismo y estado superior de salvajismo. A su vez, la siguiente etapa, barbarie, es también dividida por Morgan en otras tres etapas: estado inferior de barbarie, estado medio de barbarie y estado superior de barbarie. La última etapa es la denominada civilización, culmen de la evolución humana. A continuación se explica de forma breve las diferentes etapas 
por las que pasa la humanidad, según

Morgan detalla en Ancient Society:

I. Estadio inferior del salvajismo: desde la infancia del género humano al comienzo del período que le sigue.

II. Estadio medio del salvajismo: desde la adquisición de una subsistencia a base de pescado y el conocimiento del uso del fuego, hasta etc.

III. Estadio superior del salvajismo: desde la invención del arco y la flecha, hasta etc.

IV. Estadio inferior de la barbarie: desde la invención del arte de la alfarería, hasta etc.

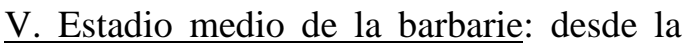
domesticación de animales en el hemisferio oriental, y en el occidental, desde el cultivo del maíz y plantas por irrigación, con el uso de adobe y piedra, hasta etc.

VI. Estadio superior de la barbarie: desde la invención de la fundición de mineral de hierro y el empleo de utensilios de hierro, hasta etc.

VII. Estadio de civilización: desde la invención de un alfabeto fonético y el empleo de la escritura, hasta el tiempo de hoy.

\section{Evolución del gobierno}

En cuanto a la evolución del gobierno, Morgan distingue dos formas de gobierno:

- Societas: una forma de organización social basada en la gens, la fratría y la tribu, propia de la sociedad antigua (salvajismo y barbarie).

- Civitas: una forma de organización política basada en el territorio y la propiedad, característica de la sociedad moderna (civilización).

El primer sistema se basa en las relaciones personales y actúa a través de la gens (linaje) y la tribu. El segundo implica la existencia de una sociedad política en la que el gobierno actúa sobre las personas a través de relaciones territoriales.

\section{Evolución de la familia}

Morgan distingue cinco estados de evolución unilineal en los diferentes períodos étnicos tratados anteriormente:

I. La familia consanguínea: institución formada sobre el matrimonio de hermanos $\mathrm{y}$ hermanas de un grupo.

II. La familia punalúa: su nombre deriva de la relación hawaiana Punalua. El matrimonio se conforma entre hermanas (o hermanos) propias y colaterales, con los maridos (o esposas) de cada una de las otras, en grupo, no siendo necesario que estuviesen emparentados entre sí. Tanto este tipo de unión como el anterior, pertenecen al estado de salvajismo.

III. La familia sindiásmica: esta institución se conforma por la unión de un hombre y una mujer, pero sin cohabitación exclusiva. Este es el origen de la familia monógama, institución 
Revista científica de la Asociación de Historia y Antropología de los Cuidados (Universidad de Alicante)

característica ya del estado de civilización.

IV. La familia patriarcal: se trata de un matrimonio de un hombre con varias mujeres

V. La familia monógama: este tipo de familia se formó por el matrimonio de un hombre con una mujer habiendo una cohabitación exclusiva. Es la institución matrimonial por la que se forma la familia de la sociedad civilizada, es decir, en la Civitas descrita con anterioridad.

\section{Niveles de subsistencia}

Morgan, argumenta que, según la escala evolutiva del ser humano, existen diferentes formas de subsistencia y de obtener alimentos desde unas formas más simples hasta unas más complejas. Los métodos simples de subsistencia corresponden a las etapas evolutivas de salvajismo. Los métodos complejos a la etapa de civilización pasando por métodos intermedios de complejidad que corresponderían con las etapas de barbarie.

I. Subsistencia natural de frutas y raíces en un hábitat restringido: subsistencia a nivel primitivo.

II. Subsistencia de pescado: se continua en el estado de salvajismo. En esta etapa se utiliza el fuego.
III. Subsistencia farinácea a través del cultivo: estado inferior de barbarie. Se comienza el cultivo de cereales y granos, aunque de forma desigual en todo el planeta.

IV. Subsistencia de carne y leche: comienza la domesticación de animales. Comienza el pastoreo como forma de vida.

V. Subsistencia ilimitada a través de la agricultura del campo: la agricultura con ayuda del ganado domesticado para cultivar y arar. Comienza una etapa en la que el nomadismo se abandona y los seres humanos se organizan en zonas geográficas determinadas pudiendo conformarse gobiernos. Esta forma de subsistencia forma parte de la civilización

El autor indica que no en todas las zonas del mundo se lograba de forma similar este progreso de subsistencia. Mientras en unas zonas se continuaba en estado primitivo o salvaje, en otras se avanzaba más en las diferentes etapas de subsistencia.

\section{REFLEXIONES FINALES}

Sin lugar a dudas, Morgan es uno de los autores más representativos del evolucionismo clásico. Dado que se interesó en primera persona por los nativos americanos, se puede decir que se diferencia un poco de sus contemporáneos como Tylor 
Cultura de los Cuidados

que eran considerados "antropólogos de sillón”. Aunque a día de hoy el método comparativo no es utilizado en antropología y algunos de sus argumentos evolucionistas se encuentren "descatalogados”, no se debe menospreciar su aporte a la disciplina y su estudio profundo que dio lugar a la antropología del parentesco y a la ecología y materialismo cultural dando aportaciones a autores de después de la Segunda Guerra Mundial como Marvin Harris.

De cara a enfermería y a antropología, he de destacar que bajo mi punto de vista tiene un remoto antecedente para comenzar a realizar investigación de campo. Como he mencionado, a diferencia de otros evolucionistas de su época, se interesó de primera mano por el grupo humano que estaba estudiando por tanto creo que se puede considerar como influyente remoto para realizar trabajo de campo. Creo que su aportación a enfermería de forma remota ha sido importante para realizar trabajo de campo, etnografía y observación participante. Y no olvidemos que acercarnos al "otro" nos hace comprender que "el otro" no es un ser extraño sino un ser humano como nosotros que comprende el mundo en sus términos y contextos culturales. La aplicación de la etnografía y de la competencia cultural no se debe dejar a un lado en este presente mundo.

\section{BIBLIOGRAFÍA}

Bohannan, P., \& Glazer, M. (2007). Lecturas de Antropología. Madrid: Mc Graw Hill.

Marrero González, C. M. (2013). Competencia Cultural. Enfoque del modelo de Purnell y Campinha-Bacote en la práctica de los profesionales sanitarios. ENE, Revista de Enfermería, 7(2).

Moreno Feliu, P. (2014). De lo Lejano a lo Próximo: un Viaje por la Antropología y sus Encrucijadas. Univ. Ramón Areces-UNED. Madrid.

Spector, R.E. (2016). Cultural Competence. Cultura de los Cuidados (Edición digital) (20)44. 9-14. Recuperado de http://dx.doi.org/10.14198/cuid.2016.44.01

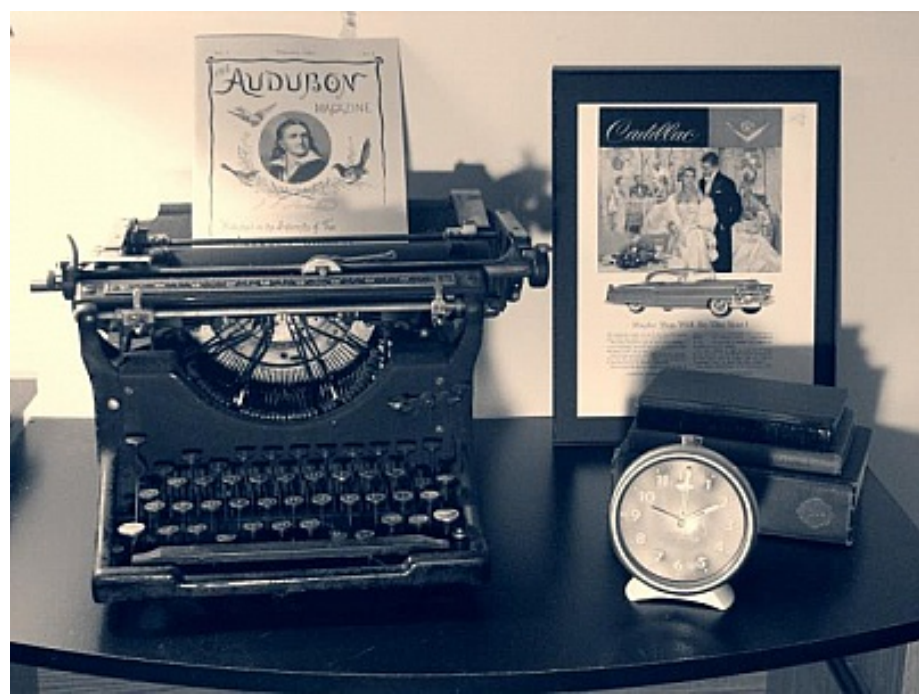

Fuente: https://freerangestock.com/photos/4754/vintage-still-life.html 